\title{
Discovery \& Proofs of a Nucleus of Mass and Charge in the Photons/Quantum Particles
}

\author{
Narendra Swarup Agarwal \\ Gurgaon, India \\ Email: nsagarwal@gmail.com
}

How to cite this paper: Agarwal, N.S. (2018) Discovery \& Proofs of a Nucleus of Mass and Charge in the Photons/Quantum Particles. Journal of Modern Physics, 9, 2170-2192.

https://doi.org/10.4236/jmp.2018.912137

Received: August 30, 2018

Accepted: October 12, 2018

Published: October 15, 2018

Copyright $\odot 2018$ by author and Scientific Research Publishing Inc. This work is licensed under the Creative Commons Attribution International License (CC BY 4.0).

http://creativecommons.org/licenses/by/4.0/

\begin{abstract}
This work discovers and proves that all the photons/quantum particles have a "Nucleus of mass and charge." The nucleus is located off-center in the particles. The constructive, destructive and intermediate Interference phenomena decisively discover and prove the presence of only one nucleus of the mass located off-center in a photon, like an atomic nucleus. The mass in the nucleus of a photon develops varying forces to move the photon as a wave. The Wave-Particle Duality is the most significant proof of such a nucleus of mass. The formation of an electromagnetic wave by a photon proves the presence of a charge in the nucleus of the photon. The Quantum Theory, developed about 100 years back, simplified the understanding of matter and energy at the atomic/subatomic levels. However, the Quantum Theory remains incomplete and cannot explain even a single quantum phenomenon. The New Quantum Theory, developed in the year 2012, is based on the similarity of an atomic nucleus as well as the solar system with the Sun as its nucleus of the mass and the charge. The New Quantum Theory explains all the Quantum Phenomena and matches with the Classical Mechanics as well as the Theory of Electromagnetism. The experiments described in this work, using high precision instruments, determine the mass, the charge and the diameter of a photon/quantum particle.
\end{abstract}

\section{Keywords}

Nucleus, Off-Center Location, Mass, Acceleration, Force and Charge

\section{Introduction}

Everything around us consists of very tiny atoms. Every atom has a nucleus of the mass $>99.9 \%$ occupying only negligibly small volume. The nucleus of an atom is not in the center of the atom but located off-center. Similarly, the solar system has the Sun as its nucleus having $>99.86 \%$ mass and occupying only neg- 
ligibly small volume. The solar system also has the Sun in the off-center position. The Sun is the most significant source of electromagnetic radiations.

Based on the above facts of nature, the New Quantum Theory [1] states:

"A photon/quantum particle has a nucleus of the mass and the charge located off-center."

A nucleus of the mass and the charge, located off-center in the spinning photon/quantum particle, bestows unique characteristics and displays strange behaviours. This constitutional essence of a photon/quantum particle explains not only the Wave-Particle Duality but all the Quantum Phenomena and completes the Quantum Theory.

The New Quantum Theory can also explain the phenomenon such as "Raman Effect" with the additional information about the external forces (varying forces in the molecular bond of a compound). The "Multiple Slits in Series" experiments describe the procedure to determine the mass, the charge and the diameter of a photon. The physical energies of a photon calculate the mass of the photon by the formula $m=a f$ where " $a$ " is a constant.

Unlike the Quantum Theory which does not match with the Classical Mechanics, the New Quantum Theory is consistent with the Classical Mechanics as well as the Theory of Electromagnetism. Moreover, the New Quantum Theory can also predict the interactions of the photons/quantum particles with other objects at the quantum level for the new developments in the scientific and commercial segments including the improvements in the PV cells.

\section{Hidden Mysteries of the Photons}

All the photons/quantum particles display mysterious phenomena known for the last several centuries. For these fundamental particles, only nature provides the hints to solve their mysteries.

A mysterious particle of the photon has its mysteries hidden inside. The following characteristics of the photons and their analysis discover the mysteries hidden within a photon:

- A photon never travels in a straight line; it moves like a helix/a smooth space curve in all the three planes or as a wave in a single two-dimensional plane.

- A photon travels with an average speed of light in the direction of its travel, irrespective of its frequency and the amplitude of the wave it forms.

- A photon always spins in one plane only. This plane does not change unless and until an external force or object interacts with the photon. A polarised photon keeps on spinning in the same plane and can travel hundreds of light years.

- A photon continuously changes its velocity and the direction to move as a wave without any external force. Whereas, according to Newton's laws of motion, a force is necessary to change the velocity and the direction of a particle.

- Therefore, a photon must develop the "Internal Forces" from within in all the $3 \mathrm{X}, \mathrm{Y} \& \mathrm{Z}$-directions to form a helix or the forces only in 2-directions to form a wave in a single two-dimensional plane. 
- The magnitudes of these internal forces in the different directions change continuously with the spin of the photon to move the photon as a wave.

- A mass generates the force on acceleration. For a photon to generate variable internal forces, it must have a "mass" along with accelerations in the different directions.

- Since the mass of the photon is constant, the acceleration/deceleration in the different directions must vary continuously to generate variable internal forces in the different directions to move the photon as a wave.

- A spinning photon with uniformly distributed mass or the mass in the center cannot develop internal forces to move the photon as a wave.

- However, if a photon has its mass in an off-center position, the mass rotates at a constant linear speed with the spin of the photon around the center of the photon. The continuous rotation of the mass develops acceleration/deceleration in the different directions continuously.

- Therefore, the mass in the spinning photon experiences variable accelerations/decelerations and develops variable internal forces in the different directions.

- Only the presence of a mass, located off-center in a photon, develops the variable forces in the different directions by accelerating/decelerating with the spin of the photon. These internal forces, created by the mass of a photon, move the photon as a wave.

- Similarly, a charge located off-center in the spinning photon accelerates/decelerates and generates the electric and the magnetic fields of the varying intensities and directions to form an electromagnetic wave.

- Both the mass and charge coexist in a so-called nucleus located off-center in the photon. There is nothing unique about the existence of such a nucleus in the photons; all the atoms have a similar nucleus of the mass and the charge.

- Similarly, all the quantum particles must also have a nucleus of the mass and the charge located off-center. An electron has a definite mass, always moves as a wave and forms an electromagnetic wave due to its charge. An electron has to have both the mass and the charge located off-center in a nucleus to travel as a wave and generate a3 dimensional electromagnetic wave.

- A photon/quantum particle spins in the plane consisting the circular path of the nucleus and the center of the photon. The nucleus of the mass and the charge rotates in this plane only.

- It appears that all the tiny particles in the universe have a nucleus of the mass and the charge located off-center. Exceptions, if any, are rare.

The above peculiarities of a photon indicate the presence of a nucleus of the mass and the charge located off-center in the photon, as stated in the New Quantum Theory.

Figure 1 shows a spinning photon as a yellow sphere with a small nucleus of mass and charge located off-center as a red sphere along with an enlarged view of the central photon. As the photon spins, the nucleus rotates in a circular path around the center of the photon. At the origin $(0,0)$, the nucleus is at $0^{\circ}$ from 


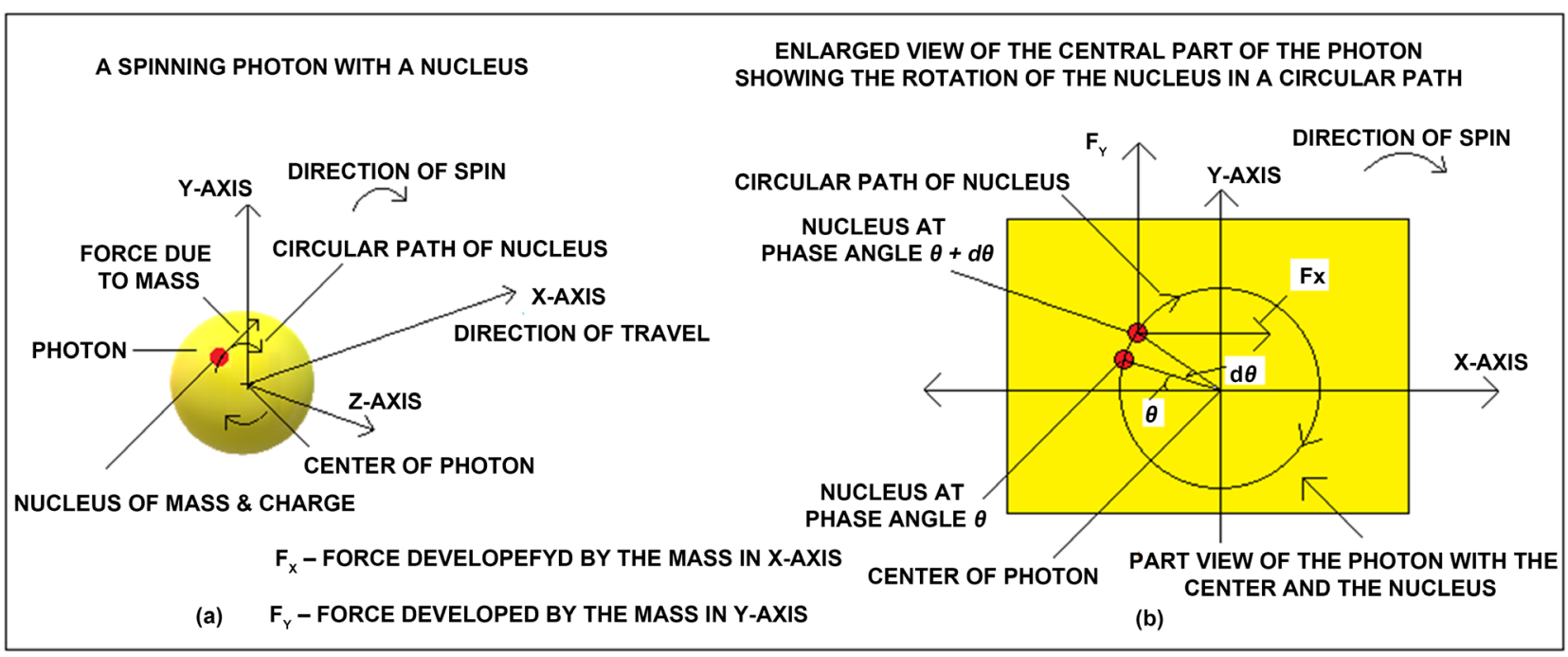

Figure 1. Shows a spinning photon as a yellow sphere with a small nucleus of mass and charge located off-center as a red sphere. As the photon spins, the nucleus rotates in a circular path around the center of the photon. Figure 1(a) shows a complete photon, and Figure 1(b) shows an enlarged view of only the central part of the photon with a red nucleus rotating around the center of the photon from the phase angle $(\theta)$ to $(\theta+d \theta)$ in time $(d t)$. The phase angle $(\theta)$ of the nucleus (or the photon) is the angular position of the nucleus in the photon from $\mathrm{X}$-axis with center of the photon as reference.

the direction of travel of the photon (X-axis) with center of the photon as the reference point. The phase angle or the angular position of the nucleus from the $\mathrm{X}$-axis increases from $0^{\circ}$ to $360^{\circ}$ with the spin of the photon in a single wave cycle. From $360^{\circ}$, the next cycle of the wave of the photon starts again from $0^{\circ}$. Figure 1(a) shows a complete photon, and Figure 1(b) shows an enlarged view of only the central part of the photon with a red nucleus rotating around the center of the photon from the phase angle $(\theta)$ to $(\theta+d \theta)$ in time $(d t)$ and developing the accelerations and the forces.

A spinning photon develops the following forces:

\section{a) Mechanical Forces:}

If the angle of the nucleus is $\theta_{1}$ from $\mathrm{X}$-axis and the photon spins from the phase angle $\theta_{1}$ to $\theta_{1}+d \theta_{1}$ in time $d t$, the force in $\mathrm{X}$-axis develops as under:

- The circumference of the rotating Nucleus: $2 \pi r$

- The linear speed of the rotating Nucleus: $2 \pi r f$

- The velocity of the nucleus in the $\mathrm{X}$-axis at phase angle $\theta_{1}: 2 \pi r f \sin \theta_{1}$

- The velocity of the nucleus in $\mathrm{X}$-axis at phase angle $\theta_{1}+d \theta_{1}$ : $2 \pi r f \sin \left(\theta_{1}+d \theta_{1}\right)$

- The change in velocity in X-axis in time $d t .2 \pi r f\left\{\sin \left(\theta_{1}+d \theta_{1}\right)-\sin \theta_{1}\right\}$

- Acceleration in X-axis: $2 \pi r f\left\{\sin \left(\theta_{1}+d \theta_{1}\right)-\sin \theta_{1}\right\} / d t$

- The force developed by the mass in the $\mathrm{X}$-axis:

$$
2 \pi r f m\left\{\sin \left(\theta_{1}+d \theta_{1}\right)-\sin \theta_{1}\right\} / d t
$$

If the phase angles of the nucleus in the photon are $\theta_{2} \& \theta_{3}$ in $\mathrm{Y}$-axis $\& \mathrm{Z}$-axis respectively, the mass in the nucleus of the photon develops the following forces:

- The force developed by the Nucleus: 


$$
2 \pi r f m\left\{\sin \left(\theta_{2}+d \theta_{2}\right)-\sin \theta_{2}\right\} / d t
$$

In the $\mathrm{Y}$-axis

- The force developed by the Nucleus:

$$
2 \pi r f m\left\{\sin \left(\theta_{3}+d \theta_{3}\right)-\sin \theta_{3}\right\} / d t
$$

In the Z-axis

where:

- The frequency of the photon: $f$

- Mass of the photon: $m$

- The distance of the nucleus from the center: $r$

- The phase angle of the photon: $\theta$

- Incremental change in phase angle: $d \theta$ degree in time $d t$

Therefore, the mass in the nucleus of a spinning photon develops the forces of the variable magnitudes in all the three directions. Depending on the phase angle $\theta$, the directions of these forces reverse in each cycle of the wave.

In case of a linearly polarised photon, the forces develop in only two axes. If $\theta$ is the phase angle from $\mathrm{X}$-axis, the forces develop as under:

- The force developed by the Nucleus:

$$
2 \pi r f m\{\sin (\theta+d \theta)-\sin \theta\} / d t
$$

in the horizontal X-axis

- The force developed by the nucleus:

$$
2 \pi r f m\{\cos (\theta+d \theta)-\cos \theta\} / d t
$$

in vertical Y-axis

The velocities, accelerations and the internal forces of the photon in the different directions vary with the values of $\sin \theta \& \cos \theta$ in the range between +1 to $(-) 1$ with the spin of the photon from $0^{\circ}$ to $360^{\circ}$ to complete one cycle of the wave.

\section{b) Electromagnetic Force:}

As a photon spins and moves, the nucleus of the mass and the charge located off-center accelerates/decelerates. Any moving charge generates both the electric as well as the magnetic fields. The charge in the photon generates the electromagnetic force along with the electromagnetic wave with the varying intensities and the changing directions.

A nucleus of the mass $\&$ the charge located off-center in a photon/quantum particle generates the mechanical forces as well as the electromagnetic forces.

\section{Mass \& Energy of the Photons-The Facts \& the Myth}

\section{THE FACTS:}

- The mass and energy always exist together.

- The energy is only the "quantitative property" of a matter. No form of energy can exist without an object of mass. Any form of energy needs an object of mass to hold the energy.

- Similarly, every object of mass has some form of the energy. 
- A photon has its mass which is always constant. A photon possesses energy in various physical forms in addition to its mass.

- Planck's equation $\boldsymbol{E}=\boldsymbol{h} f$ relates to the physical forms of energies of a photon.

- The mass of a photon is proportional to its frequency. Equation $\boldsymbol{m}=\boldsymbol{a} \mathrm{f} \mathrm{cal}-$ culates the "Mass $(m)$ " of the photon of frequency $f$, where " $a$ " is constant ( $a$ $=1.474499440284 \times 10^{-50} \mathrm{~kg} \cdot \mathrm{sec}$ ) [2].

- Einstein's equation $\boldsymbol{e}=\boldsymbol{m} \boldsymbol{c}^{2}$ relates to the conversion of energy to mass and vice versa involving the nuclear conversion. This energy " $e$ " is in addition to the physical forms of the energy's " $E$ " possessed by the photon. Both the forms of the energies " $e$ " and " $E$ " relate to the different types of the energies.

- The mass of a photon can be calculated theoretically as well as determined by the experiments using high precision instruments.

- A nucleus of the mass and the charge exists in an off-center position and rotates around the center of the spinning photon. The nucleus of the mass and the charge continuously accelerates and decelerates to create the variable mechanical forces and the electromagnetic forces to exhibit all the quantum phenomena.

THE MYTH:

- The Photons have no mass \& no charge.

The above myth must go.

\section{Theoretical Proofs}

All the Quantum Phenomena are the proofs and can only take place if a photon has a "nucleus of mass and charge, located off-center." Some of the important Quantum Phenomena as the proof of the presence of a nucleus of mass and the charge in a nucleus located off-center in the photons are presented below:

\subsection{Polarisation Phenomenon}

In the polarisation phenomenon, a photon/quantum particle forms a wave in a single two-dimensional plane. The continuously accelerating mass develops forces in a single two-dimensional plane to form a wave by a polarized photon. The following characteristics describe the Polarisation Phenomenon:

- A critical feature of a photon is: "A photon spins only in one plane and continues to spin in the same plane unless it interacts with an external force/object."

- If a photon/quantum particle is a uniform particle with zero mass, there is nothing to restrict the plane of the spin of the photon and the formation of the wave in one single plane only. The photon is always at the liberty to keep on changing the plane of spin and to form the waves in the different planes, in such a case, the polarisation phenomenon for a photon with zero mass cannot take place.

- Only the forces, developed in a single two-dimensional plane by the mass in the nucleus located off-center in a photon/quantum particle, limit the photon particle to form a wave in a single two-dimensional plane and display the pola- 
risation phenomenon. The direction of travel of a linearly polarized photon confines in the plane of the circular path of the nucleus/spin of the photon.

- The axis of rotation of the nucleus or the spin of the photon is the same and always perpendicular to the direction of travel of the polarised photon.

- As a polarised photon spins and travels in X-direction, the mass in the nucleus located off-center accelerates/decelerates to develop variable forces only in $\mathrm{X} \& \mathrm{Y}$-directions. These forces, from within the photon, form a wave in a single two-dimensional plane.

- Figure 2 shows a polarised photon developing the variable forces $F_{X}$ and $F_{Y}$ with the spin of the photon to form a wave in a two-dimensional X, Y plane. The one full wave cycle of the photon shows the position of the nucleus as a small red sphere at different phase angles with the spin of the photon starting from $0^{\circ}$ to $360^{\circ}$ with an interval of $45^{\circ}$ along with the magnitudes of the forces $\mathrm{F}_{\mathrm{X}}$ and $\mathrm{F}_{\mathrm{Y}}$ being indicated by the size of the arrows approximately.

- The electric field wave of a polarised photon forms in a single two-dimensional plane; but the magnetic field wave always forms in the three-dimensional planes.

There are two types of polarisation phenomena:

- Linear Polarisation Phenomenon:

Already explained above.

\section{- Circular Polarisation Phenomenon:}

If the direction of travel of a photon is outside the plane of its spin, the photon is circularly polarised. For a circularly polarised photon, the axis of rotation of the photon is not perpendicular to the direction of travel of the photon.

A circularly polarised photon moves as a smooth curve in three-dimensional space/a helix. The curve may be either clockwise or anticlockwise.

Whereas, the photon spins in the plane consisting the nucleus of the mass and the center of the photon, the circularly polarised photon travels in a direction outside this plane. Therefore, the whole photon along with the nucleus of the mass moves in all the $3 \mathrm{X}, \mathrm{Y} \& \mathrm{Z}$-directions and creates variable internal forces $\mathrm{F}_{\mathrm{X}}, \mathrm{F}_{\mathrm{Y}} \& \mathrm{~F}_{\mathrm{Z}}$ in all the $\mathrm{X}, \mathrm{Y} \& \mathrm{Z}$-directions respectively.

These variable internal forces in $\mathrm{X}, \mathrm{Y} \& \mathrm{Z}$-directions move the photon to form a wave in the three-dimensional plane.

The Polarisation Phenomenon discovers and proves the presence of a mass located off-center in a photon.

\subsection{Interference}

"Interference" is the most critical phenomenon of the Quantum Physics. The Interference phenomenon discovered the Dual Wave-Particle nature of the photon's way back in the year 1801 by the Double Slit experiment.

- The two photons of the same frequency superpose in the different phases to display different types of Interferences with different amplitudes of the resultant waves. 




Figure 2. The mass in the nucleus of a spinning photon rotates around the center of the photon and generates forces of varying magnitudes in $\mathrm{X}$-axis as well as $\mathrm{Y}$-axis to form a wave in a single two-dimensional $\mathrm{X}-\mathrm{Y}$ plane. The wave shows the direction of the forces $\mathrm{F}_{\mathrm{X}}$ and $\mathrm{F}_{\mathrm{Y}}$ at different phase angles of the photon.

- The phase angle of a photon refers to the angle of its nucleus from the direction of travel (generally, the X-axis) with the center of the photon as the reference. At the start of a new wave cycle or the origin, the phase angle/angular position of the nucleus or the photon is $0^{\circ}$. As the photon spins, the nucleus of the mass and the charge rotates around the center of the photon, and the phase angle increases from $0^{\circ}$ up to $360^{\circ}$ and from the $360^{\circ}$ a new wave cycle of the photon starts.

- If the photons are uniform particles with no substructure, whatever are their orientations or the phase angles or the degree of spins, all the photons remain identical with no difference. Therefore, all the photons of the same frequency must display the same character/properties in all the $360^{\circ}$ spins or the phase angles, and the phase angles or the degree of the spin of the photons has no meaning.

- In the above situation of the photons as the uniform particles, the different types of the Interference Phenomena are not possible.

- The phase angle or the degree of spin of a photon from $0^{\circ}$ to $360^{\circ}$ refers to the angular position of a substructure in the photon. This proves the presence of a substructure in a photon.

- The $0^{\circ}$ phase angle of a photon indicates the position of the nucleus (substructure of the photon) at $0^{\circ}$ angular position from the $\mathrm{X}$-axis with the center of the photon as the reference. At the $0^{\circ}$, the photon is at the origin $(0,0)$ or the start point of a new cycle of the wave.

- As the photon spins and forms a wave the nucleus of the mass and the charge located off-center rotates around the center of the photon and the phase an- 
gle or the angular position of the nucleus increases from $0^{\circ}$ to $360^{\circ}$ in the photon in one wave cycle of the wave.

- The two photons of the same frequency can superpose in the different phases to display different types of the Interference effects.

If the two photons of the same frequency and in the same direction superpose, the two photons can join only in side by side position. Both the superposed photons keep on spinning in their direction of spin and move together. The mass in each photon being close enough, the strong force is applied to keep the superposed photons together. Figure 3 shows the two spinning photons joined side by side.

\subsubsection{Constructive Interference}

In the Constructive Interference of the two photons, the amplitude of the resultant wave doubles. When the two photons of the same frequency and in the same phase superpose, the angular positions of the nuclei of both the photons are also the same as shown in the part (a) of Figure 4. The mass, in the nucleus of both the photons, accelerates/decelerates and develops the forces of the same magnitude all the time. The force $\mathrm{F}_{\mathrm{Y}}$ in the vertical direction, developed by the mass of each photon, adds to double as under:

- The force developed by the nucleus of: $2 \pi r f m\{\cos (\theta+d \theta)-\cos \theta\} / d t$

Photon No. 1 in Vertical Y-axis

- The force developed by the nucleus of: $2 \pi r f m\{\cos (\theta+d \theta)-\cos \theta\} / d t$

Photon No. 2 in Vertical Y-axis

- Total Force developed by the nuclei of: $4 \pi r f m\{\cos (\theta+d \theta)-\cos \theta\} / d t$

Photons 1 \& 2 in Vertical Y-axis

If a photon has zero mass, it cannot develop any force to double the amplitude.

Only the presence of a mass in each photon generates the vertical forces in both the photons to double the amplitude. The phenomenon of Constructive Interference' with the double amplitude of the resultant wave discovers and proves: " $A$ Photon has Mass."

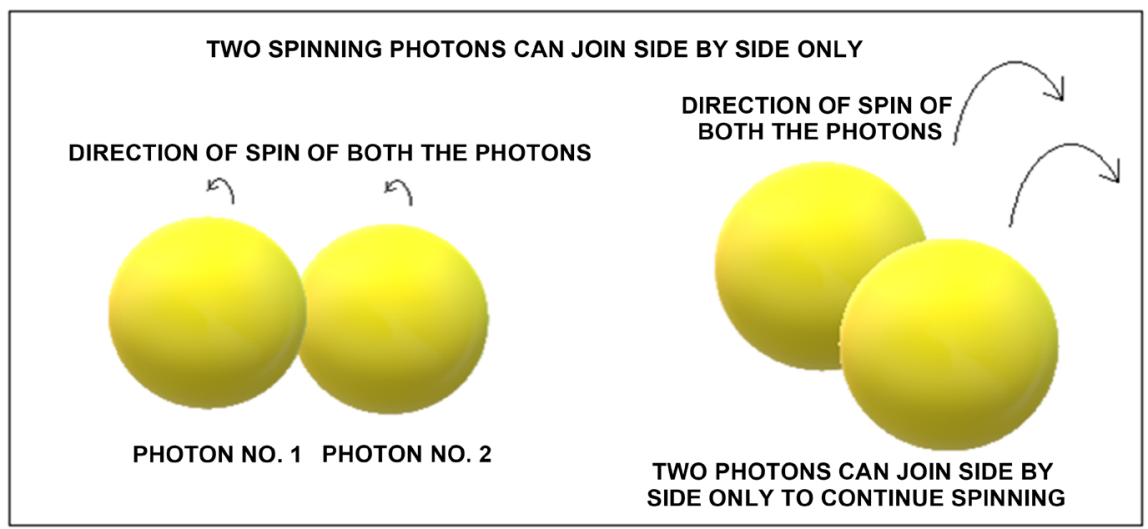

Figure 3. Two same frequency spinning photons moving in the same direction can join in side by side position only so that both the photons continue spinning in the same direction. 




Figure 4. Part (a) shows the two photons in the same phase developing forces of equal magnitudes in the same direction. Part (b) shows the two photons in the opposite phase developing forces of equal magnitudes in the opposite directions. Part (c) shows the two photons developing forces of the different magnitudes and directions.

Even though the Constructive Interference proves the presence of the mass in the photons, it does not indicate the location of the mass in the photon.

\subsubsection{Destructive Interference}

In the Destructive Interference, the two photons superpose with a phase difference of $\pi$ and form the resultant wave with the zero amplitude. The part (b) of Figure 4 shows the two photons of the same frequency with a phase difference of $\pi$ in the superposed position developing the forces of equal magnitudes in the opposite directions.

The nuclei of the mass of both the spinning photons have a phase angle difference of $\pi$. The directions of the forces developed by the mass in the nucleus of each photon are always opposite to each other with a phase difference of $\pi$. If the phase angle of the Photon No. 1 is $(\theta)$ and the phase angle of the Photon No. 2 is $(\theta+\pi)$. The two photons develop forces in the vertical direction as under:

- The force developed by the nucleus of: $2 \pi r f m\{\cos (\theta+d \theta)-\cos \theta\} / d t$

Photon No. 1 in Vertical Y-axis

- The force developed by the nucleus of:

$$
2 \pi r f m\{\cos (\theta+\pi+d \theta)-\cos (\theta+\pi)\} / d t
$$

Photon No. 2 in Vertical Y-axis

$$
\text { Or: }-2 \pi r f m\{\cos (\theta+d \theta)-\cos \theta\} / d t
$$

- The resultant force developed by the nuclei: Zero

Of both the Photons in Vertical Y-axis

Since the net vertical force of both the photons is always zero, the amplitude of the resultant wave is also always the zero. The Destructive Interference phenomenon is not possible if the mass is uniformly distributed or in the center of the photon. In such a situation, whatever is the phase difference between the two photons, only the Constructive Interference can take place. 
The Destructive Interference is possible only in a condition: "The location of the nucleus of the mass is in the off-center position in the photons." However, the Destructive Interference phenomenon can also take place even if a photon has more than one nucleus of the mass, located off-center in the photon.

The phenomenon of Destructive Interference with the zero amplitude of the resultant wave discovers and proves: " $\underline{A}$ photon has the mass located in an off-center position."

\subsubsection{Intermediate Interference}

When the two photons, with a phase difference other than $0^{\circ}$ or $\pi$, superpose the resultant wave with the amplitude $>0$ but $<$ double the amplitude forms due to the phenomenon of the Intermediate Interference. Figure 3(c) shows such two interfering photons.

If the two photons of the same frequency with phase angles $\theta_{1} \& \theta_{2}$ interfere, the mass in the nucleus of each photon develops the force in the vertical direction as under:

- The force developed by the nucleus of: $2 \pi r f m\left\{\cos \left(\theta_{1}+d \theta_{1}\right)-\cos \theta_{1}\right\} / d t$ Photon No. 1 in Vertical Y-axis

- The force developed by the nucleus of: $2 \pi r f m\left\{\cos \left(\theta_{2}+d \theta_{2}\right)-\cos \theta_{2}\right\} / d t$ Photon No. 2 in Vertical Y-axis

- Total force developed by the nuclei of both the Photons in Vertical Y-axis:

$$
2 \pi r f m\left\{\cos \left(\theta_{1}+d \theta_{1}\right)-\cos \theta_{1}\right\} / d t+2 \pi r f m\left\{\cos \left(\theta_{2}+d \theta_{2}\right)-\cos \theta_{2}\right\} / d t
$$

The amplitude of the resultant wave depends on the difference in the phase angles $\theta_{1} \& \theta_{2}$ of the two photons. If a photon has more than one nucleus of the mass, the two photons on intermediate interference must form a resultant wave with two or more peaks. However, the resultant wave has only a single peak (there is no reference of the resultant wave with the Intermediate Interference of more than one peak). The single peak of the resultant wave confirms the presence of only one nucleus of the mass located off-center in a photon.

The phenomenon of Intermediate Interference produces the resultant wave with the sum of the amplitudes of both the photons discovers and proves:

\section{"A photon has only one nucleus of the mass located in the off-center posi-} tion."

Note: The horizontal forces developed by the mass in the nucleus of the photons are not required here to prove the presence of only one nucleus of the mass located off-center in the photons.

\subsection{Wave-Particle Duality}

The Dual Wave-Particle nature of a photon awaits explanation since the year 1801. A particle of a photon/quantum always moves like a wave and displays the properties of the particle as well as the wave. Only the New Quantum Theory explains the Wave-Particle Duality. The followings describe the characteristics of a linearly polarised photon traveling in the horizontal $\mathrm{X}$-axis and forming a wave 
in X-Y plane:

- A photon particle continuously changes its velocity and direction to move as a wave.

- The photon spins and completes one cycle of the wave in $360^{\circ}$ spins with half the wave above the line of the travel and the other half below. Both the halves have a single peak.

- For a wave starting from the origin $(0,0)$, the photon moves up with the maximum vertical velocity in the $\mathrm{Y}$-axis which reduces to zero at $90^{\circ}$ phase angle. The photon descends from $90^{\circ}$ phase angle achieving the maximum downward velocity at $180^{\circ}$ phase angle but this velocity again turns to zero at $270^{\circ}$ phase angle, and from there the photon ascends.

- In addition to the speed of light in the horizontal X-axis, the mass in the nucleus of the photon also develops the acceleration/velocity in the horizontal direction. Therefore, the horizontal velocity of a photon varies continuously in a wave cycle with the speed of light being always the average in a single wave cycle.

- The horizontal velocity of the photon (due to the mass in the nucleus) is zero at the origin, the maximum at $90^{\circ}$ phase angle, again zero at $180^{\circ}$ phase angle and the maximum in the reverse direction at $270^{\circ}$ phase angle.

- The up and down cyclical movement of a photon to form a wave is only possible by the presence of a mass located off-center in the photon. The mass, in the nucleus of a photon located off-center, continuously accelerates/decelerates to produce variable forces in both the horizontal $\mathrm{X}$-axis and the vertical Y-axis.

The following is the science of the "Formation of a wave by a particle of a linearly polarised photon":

- The horizontal velocity of a photon at phase angle $\theta .2 \pi r f \sin \theta+c$

- The force developed by the Nucleus: $2 \pi r f m\{\sin (\theta+d \theta)-\sin \theta\} / d t$

in the horizontal X-direction

- The vertical velocity of a photon at phase angle $\theta \cdot 2 \pi r f \cos \theta$

- The force developed by the nucleus: $2 \pi r f m\{\cos (\theta+d \theta)-\cos \theta\} / d t$ in the vertical Y-direction

where, " $c$ " is the average speed of light in $\mathrm{X}$-axis.

The horizontal velocity and the force in the horizontal direction are the functions of $\sin \theta$. The vertical velocity and force in the vertical direction are the functions of $\cos \theta$. With the spin of the photon from $0^{\circ}$ to $360^{\circ}$ phase angle, the values of $\sin \theta$ and $\cos \theta$ change between +1 and $(-) 1$. For the same frequency photons, the values of $r, f \& m$ are constant. The internal forces, developed from within the photon, move the photon as a wave as under:

\section{A) Formation of $1^{\text {st }}$ Quarter of Wave: Phase Angle from $0^{\circ}$ to $90^{\circ}$ :}

- At $0^{\circ}$ phase angle or the origin of a new wave cycle, the value of $\operatorname{Sin} \theta$ is zero. Therefore, the horizontal velocity (due to the mass in the photon) is also zero. In this quarter as the photon spins and moves from $0^{\circ}$ to $90^{\circ}$ phase angle, 
the value of $\sin \theta$ increases from 0 to +1 .

- The horizontal velocity and the horizontal force change from $0^{\circ}$ to $90^{\circ}$ as under:

The horizontal velocity: Zero at $0^{\circ}$ phase angle and the maximum at $90^{\circ}$

The horizontal force: The maximum force at $0^{\circ}$ phase angle and reduces to zero at $90^{\circ}$

- At $90^{\circ}$ phase angle, the value of $\sin \theta$ is 1 . Therefore, the horizontal velocity at $90^{\circ}$ phase angle grows to the maximum.

- At $0^{\circ}$ phase angle or the origin of a new wave cycle, the value of $\cos \theta$ is +1 . Therefore, its vertical velocity (due to the mass in the photon) is the maximum. In this quarter as the photon spins and moves from $0^{\circ}$ to $90^{\circ}$ phase angle, the value of $\cos \theta$ decreases from +1 to 0 .

- The vertical velocity and the vertical force change from $0^{\circ}$ to $90^{\circ}$ as under:

The vertical velocity: The maximum at $0^{\circ}$ phase angle and reduces to zero at $90^{\circ}$

The vertical force: Zero at $0^{\circ}$ phase angle and increases to the maximum at $90^{\circ}$ in $(-) \mathrm{Y}$-axis

- At $90^{\circ}$ phase angle, the value of $\cos \theta$ is zero. Therefore, the vertical velocity at $90^{\circ}$ phase angle turns to zero, and the photon cannot move further up anymore in the $\mathrm{Y}$-axis from the phase angle $90^{\circ}$. The photon reaches the highest point of the wave at $90^{\circ}$ phase angle.

B) Formation of $2^{\text {nd }}$ Quarter of Wave: Phase Angle from $90^{\circ}$ to $180^{\circ}$ :

- At $90^{\circ}$ phase angle, the value of $\sin \theta$ is +1 with the maximum horizontal velocity. In this quarter as the photon spins and moves from $90^{\circ}$ to $180^{\circ}$ phase angle, the value of $\sin \theta$ decreases from +1 to 0 .

- The horizontal velocity and the horizontal force change from $90^{\circ}$ to $180^{\circ}$ as under:

The horizontal velocity: The maximum at $90^{\circ}$ phase angle and reduces to zero at $180^{\circ}$

The horizontal force: Zero at $90^{\circ}$ phase angle and increases to the maximum at $180^{\circ}$ in $(-) \mathrm{X}$-axis

- At $180^{\circ}$ phase angle, the value of $\sin \theta$ is zero. Therefore, the horizontal velocity at $180^{\circ}$ phase angle (due to the mass in the photon) turns to zero.

- At $90^{\circ}$ phase angle, the value of $\cos \theta$ is zero with the zero vertical velocity. In this quarter as the photon spins and moves from $90^{\circ}$ to $180^{\circ}$ phase angle, the value of $\cos \theta$ decreases from 0 to (-) 1 .

- The vertical velocity and the vertical force change from $90^{\circ}$ to $180^{\circ}$ as under: The vertical velocity: Zero at $90^{\circ}$ phase angle and increases to the maximum at $180^{\circ}$ in $(-)$ Y-axis

The vertical force: The maximum at $90^{\circ}$ phase angle and reduces to zero at $180^{\circ}$ in $(-)$ Y-axis

- At $180^{\circ}$ phase angle, the value of $\cos \theta$ is (-) 1 with the maximum vertical velocity in (-)Y-axis and the photon completes the upper half cycle of the wave formation. From $180^{\circ}$ phase angle, the photon moves below the line of travel 
(X-axis) of the photon.

C) Formation of $3^{\text {rd }}$ Quarter of Wave: Phase Angle from $180^{\circ}$ to $270^{\circ}$

- At $180^{\circ}$ phase angle, the value of $\sin \theta$ is zero with the zero-horizontal velocity (due to the mass of the photon). In this quarter as the photon spins and moves from $180^{\circ}$ to $270^{\circ}$ phase angle, the value of $\sin \theta$ decreases from 0 to $(-) 1$.

- The horizontal velocity and the horizontal force change from $180^{\circ}$ to $270^{\circ}$ as under:

The horizontal velocity: Zero at $180^{\circ}$ phase angle and increases to the maximum at $270^{\circ}$ in $(-) \mathrm{X}$-axis

The horizontal force: The maximum at $180^{\circ}$ phase angle and reduces to zero at $270^{\circ}$ in $(-) \mathrm{X}$-axis

- At $270^{\circ}$ phase angle, the value of $\sin \theta$ is $(-) 1$ with the maximum horizontal velocity in (-)X-axis.

- At $180^{\circ}$ phase angle, the value of $\cos \theta$ is $(-) 1$ with the maximum vertical downward velocity. In this quarter as the photon spins and moves from $180^{\circ}$ to $270^{\circ}$ phase angle, the value of $\cos \theta$ increases from (-) 1 to 0 .

- The vertical velocity and the vertical force change from $180^{\circ}$ to $270^{\circ}$ as under:

The vertical velocity: The maximum at $180^{\circ}$ phase angle in (-)Y-axis and reduces to zero at $270^{\circ}$

The vertical force: Zero at $180^{\circ}$ phase angle and increases to the maximum at $270^{\circ}$ in + Y-axis

- At $270^{\circ}$ phase angle, the value of $\cos \theta$ is zero with the zero-vertical velocity; therefore, the photon cannot go down further. The photon reaches the lowest point of the wave at $270^{\circ}$ phase angle.

D) Formation of $4^{\text {th }}$ Quarter of Wave: Phase Angle from $270^{\circ}$ to $360^{\circ}$

- At $270^{\circ}$ phase angle the value of $\operatorname{Sin} \theta$ is (-) 1 with the maximum horizontal velocity (due to the mass of the photon) in the (-)X-direction. In this quarter as the photon spins and moves from $270^{\circ}$ to $360^{\circ}$ phase angle, the value of $\sin \theta$ increases from $(-) 1$ to 0 .

- The horizontal velocity and the horizontal force change from $270^{\circ}$ to $360^{\circ}$ as under:

The horizontal velocity: The maximum at $270^{\circ}$ phase angle in $(-) \mathrm{X}$-axis and reduces to zero at $360^{\circ}$

The horizontal force: Zero at $270^{\circ}$ phase angle and increases to the maximum at $360^{\circ}$ in $+\mathrm{X}$-axis

- At $360^{\circ}$ phase angle, the value of $\sin \theta$ is zero; therefore, the horizontal velocity at $360^{\circ}$ turns to zero.

- At $270^{\circ}$ phase angle the value of $\cos \theta$ is zero with the zero-vertical velocity. In this quarter as the photon spins and moves from $270^{\circ}$ to $360^{\circ}$ phase angle, the value of $\cos \theta$ increases from 0 to +1 .

- The vertical velocity and the vertical force change from $270^{\circ}$ to $360^{\circ}$ as under: The vertical velocity: Zero at $270^{\circ}$ phase angle and increases to the maximum 
at $360^{\circ}$ in $+\mathrm{Y}$-axis

The vertical force: The maximum at $270^{\circ}$ phase angle and reduces to zero at $360^{\circ}$ in $+Y$-axis

- At $360^{\circ}$ phase angle, the value of $\cos \theta$ is +1 with the maximum vertical velocity in $+\mathrm{Y}$-axis and the photon completes the lower half of the wave.

Figure 2 shows a wave formed by a linearly polarised photon spinning from $0^{\circ}$ to $360^{\circ}$ phase angle to form one full cycle of the wave. This figure also shows the forces with their directions developed by the mass in the nucleus located off-center in the photon at different phase angles or the angular position of the nucleus in the photon.

The Wave-Particle Duality is only possible if, and only if, a spinning photon has a mass located off-center in the photon and proves the presence of a mass located off-center in a photon.

If a photon has either the zero mass or uniformly distributed mass, the Wave-Particle Duality and other quantum phenomena are not possible.

\subsection{To Find the Location of a Photon in Wave at Any Phase Angle}

The following equations calculate the exact location of a photon in a wave cycle:

A photon of frequency " $f$ " spins from the phase angle $\theta$ to $\theta+d \theta$, the time and the average velocity are as under:

- The time required by a photon to spin $d \theta$ degree: $d \theta /(360 f)$

- The average horizontal velocity in $d \theta$ spin: $[c+\pi r f\{\sin \theta+\sin (\theta+d \theta)\}]$

- The horizontal distance covered in $d \theta$ spin:

$$
[c+\pi r f\{\sin \theta+\sin (\theta+d \theta)\}] d \theta /(360 f)
$$

- The average vertical velocity:

$$
\pi r f\{\cos \theta+\cos (\theta+d \theta)\}
$$

- The vertical distance covered in $d \theta$ spin:

$$
\pi r f\{\cos \theta+\cos (\theta+d \theta)\} d \theta /(360 f)
$$

The integration, of the equation nos. (6) \& (7) from the phase angle $\theta_{1}$ to $\theta_{2}$ calculates the location of the photon in a quadrant. The integration of the equation no. (7) from $0^{\circ}$ to $90^{\circ}$, calculates the amplitude of the wave formed by a photon. The above equations need calculations for each quadrant separately since the same values of $\sin \theta$, and $\cos \theta$ between $0^{\circ}$ to $360^{\circ}$ phase angle repeat at different phase angles.

For the photon of known amplitude of the wave, the equation no. (7) calculates the value of " $r$ " for the photon.

\subsection{Formation of an Electromagnetic Wave}

The following is the science of "The Formation of an Electromagnetic Wave by a photon":

- A moving point charge generates both the electric field as well as the magnetic field. 
- As the velocity of the charge increases, the intensities of both the electric and the magnetic fields increase and vice versa. If the charge moves in the reverse direction, the directions of both the electric and magnetic fields generated are also in the reverse directions.

- During the $1^{\text {st }}$ half cycle of the spin of the photon from $0^{\circ}$ to $180^{\circ}$ phase angle, the charge, in the nucleus located off-center in the photon, moves in the forward direction in comparison to the center of the photon. Figure 5 shows a spinning photon with the nucleus moving in the forward direction.

- The velocity of the charge in the nucleus in the forward direction increases from $0^{\circ}$ to $90^{\circ}$ spin and decreases from $90^{\circ}$ to $180^{\circ}$ spin of the photon or the phase angle. Therefore, the intensities of both the electric and the magnetic fields increase from $0^{\circ}$ to $90^{\circ}$ and decrease from $90^{\circ}$ to $180^{\circ}$ spin of the photon or the phase angles.

- During the $2^{\text {nd }}$ half cycle of the spin of the photon from $180^{\circ}$ to $360^{\circ}$ phase angle, the charge, in the nucleus located off-center in the photon, moves in the backward direction in comparison to the center of the photon. Figure 5 also shows a spinning photon with the nucleus moving in the backward direction.

- As the charge moves in the backward direction during $180^{\circ}$ to $360^{\circ}$ spin, now both the electric and the magnetic fields are generated in the reverse/opposite direction.

- The velocity of the charge in the nucleus (in the reverse direction) increases from $180^{\circ}$ to $270^{\circ}$ spin and decreases from $270^{\circ}$ to $360^{\circ}$ spin of the photon or the phase angle. Therefore, the intensities of both the electric and the magnetic fields increase from $180^{\circ}$ to $270^{\circ}$ and decrease from $270^{\circ}$ to $360^{\circ}$ spin of the photon or the phase angle, but in the opposite directions.

- After completing one cycle of the electromagnetic wave formation in $360^{\circ}$ spin of the photon, the new cycle of the electromagnetic wave starts again from $0^{\circ}$ phase angle.

The charge in the nucleus accelerates/decelerates to produce the electric field wave in a two-dimensional plane and the magnetic field wave in three-dimensional plane. Figure 6 shows one full cycle of the Electromagnetic Wave formed during the $0^{\circ}$ to $360^{\circ}$ spin of a polarised photon. The above describes the formation of an electromagnetic wave briefly only, as the "New Quantum Theory explains all the Mysterious Quantum Phenomena" [2] explains the formation of an electromagnetic wave by a photon with enough details.

The formation of an Electromagnetic Wave by a photon discovers and proves the presence of a charge in the nucleus located off-center in the photon.

\subsection{Refraction}

The phenomenon of refraction changes the direction and the velocity of a photon in the new medium. The secret of the refraction phenomenon are the interactions between the electromagnetic fields of both the photon as well as the new 


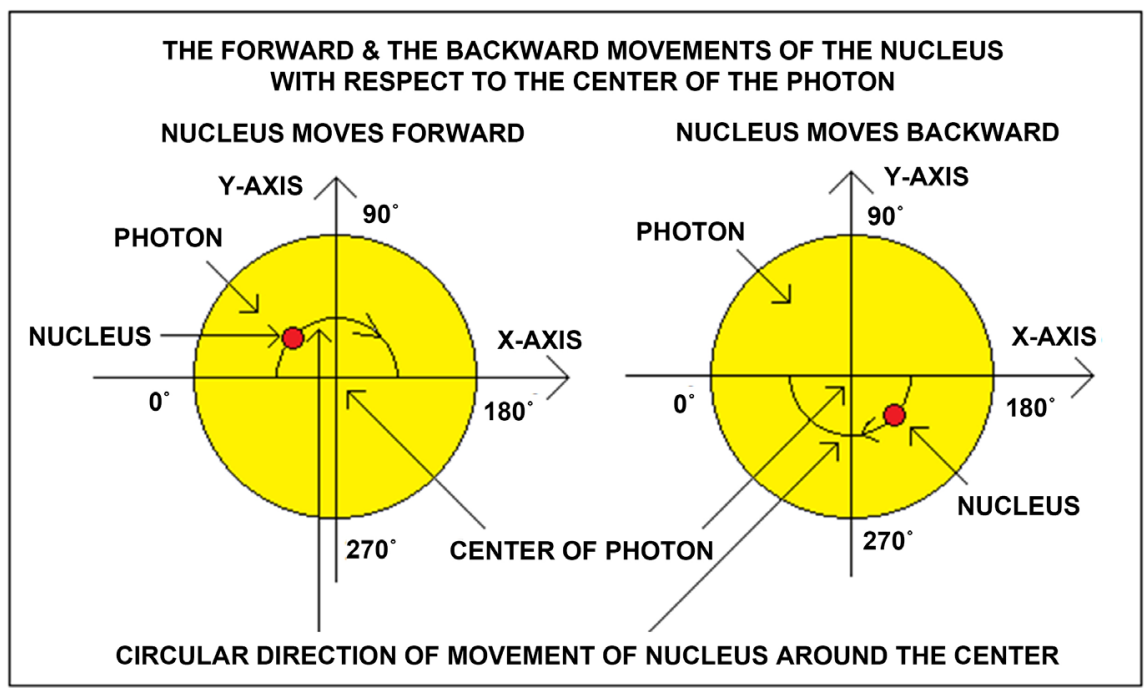

Figure 5. The nucleus of the mass circles around the center of the spinning photon. The nucleus moves in the forward direction during $0^{\circ}$ to $180^{\circ}$ phase angle with respect to center of the photon. The nucleus moves in the backward direction during $180^{\circ}$ to $360^{\circ}$ phase angle with respect to center of the photon.

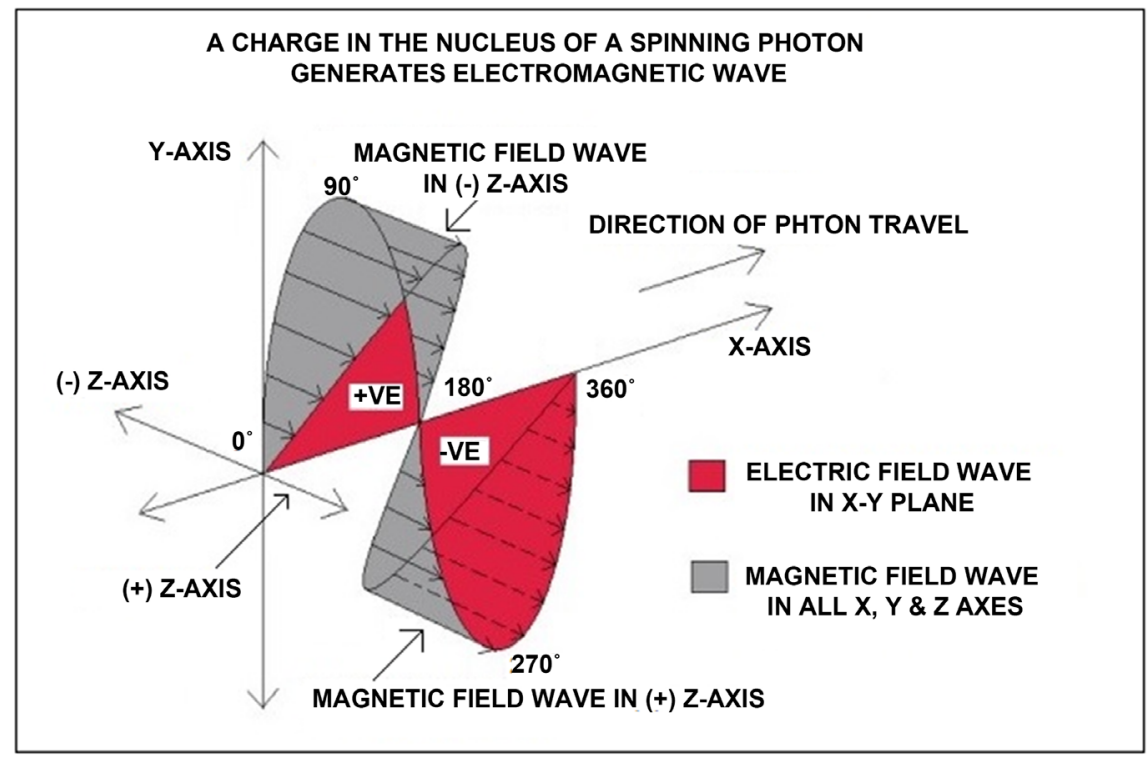

Figure 6. A charge in the off-center nucleus circles the center of photon and moves in the forward as well as the backward directions. The forward movement above the line of travel (X-axis) and backward movement below the line of travel generate the electric and the magnetic field waves in the opposite directions.

medium. A linearly polarised photon explains the phenomenon of refraction as under:

- As a rule, a photon spins in the plane of the rotation of the nucleus around the center of the photon. The mass in the nucleus of a linearly polarised photon generates the forces in the X-Y plane only and the photon travels in $\mathrm{X}$-axis. The charge in the nucleus generates the electric field in the $\mathrm{X}-\mathrm{Y}$ plane 
on both up \& down sides of the line of travel of the photon. The magnetic field generates in all the three directions on both the sides of the line of travel in the plane perpendicular to X-Y plane of the electric field. Figure 6 shows the planes of the Electromagnetic Wave formed by a linearly polarized photon.

- When a polarised photon travels in the vacuum in the absence of any external electromagnetic field, the charge in the photon generates the uniform electric field and the uniform magnetic field on both the sides of the line of travel of photon.

- A medium consists of atoms bonded together as the molecules. The molecular bonds of the medium generate their distinct electromagnetic fields.

- As the photon enters from the vacuum to the new medium, it experiences a new environment of the external electromagnetic field. Both the electromagnetic fields of the photon and the medium interact.

- A photon is an independent moving identity whereas a medium, in comparison to the photon, is a solid or liquid with a rigid structure and molecular bonds with the electromagnetic field.

- The external electromagnetic field forces the photon to tilt its plane of spin to adjusts to the new external electromagnetic field. The tilt of the photon changes the directions of its electric and the magnetic fields and finds the path of the least electromagnetic resistance to travel in the medium.

- With the tilt of the plane of spin of the photon, the nucleus of the mass and the charge rotates around the center of the photon in a new plane. The mass in the nucleus now generates the forces in the new plane and the photon now travels in a new direction matching with the plane of spin of the photon. Therefore, the direction of the travel of photon changes in the new medium.

- Figure 7 shows a photon moving from vacuum to a medium and again to the vacuum, the electromagnetic field of the medium changes the direction of the photon in the medium.

- As the photon travels out from the medium and its electromagnetic field, the photon is now out of the external magnetic field. Therefore, the photon tilts back to its regular plane of spin and direction, and all the above changes revert to the normal status.

As the photon enters a new medium and its electromagnetic field, the plane of the spin of photon tilts resulting in the following changes in the photon:

1) The photon travels in a new direction matching with the new plane of the spin of the photon as the forces developed by the mass in the nucleus are in the new directions.

2) The photon forms a wave in a new two-dimensional plane.

3) A medium always contracts the electric field of the photon in comparison to the vacuum. The reduction in the size of the electric field wave now formed by the photon reduces the wavelength of the photon in the medium. Therefore, the velocity of the photon reduces in the new medium. 




Figure 7. Shows a photon traveling from the vacuum to a medium resulting in the change of direction of travel. As the photon exits to the vacuum, the plane of spin tilts again to return to its original direction.

The phenomenon of Refraction discovers and proves the presence of a nucleus of the mass and the charge in a photon.

\section{Experiments}

When a photon reflects on a mirror, the rotating mass in the nucleus of the photon generates an inertial torque at the contact point of the mirror. This inertial torque deviates the photon very slightly in all the three axes. The new equation of reflection for a photon is as under:

$$
i=i+d
$$

where " $i$ " is the angle of incidence and " $d$ " is the angle of deviation of the photon in an axis.

This little deviation of the photons depends on the angle of incidence and the angle of polarisation of the photons. Generally, this deviation is tiny and below the noticeable limit. However, this little deviation on the reflection can be multiplied by the followings:

a) By the second reflection of the deviated photons from a circular surface coated mirror.

b) By reflecting a long chain of the photons.

By repeating the constructive interference again and again, a long chain of the photon forms. When only the $1^{\text {st }}$ photon at the beginning of the chain contacts and reflects on a mirror, the torque of all the spinning photons transfers to the contact point of the $1^{\text {st }}$ photon with the mirror. Therefore, the angle of the deviation of the whole chain of the photons multiplies in all the three axes. Figures 8 (a)-(c) shows the horizontal, inclined and the vertical chains of the photons for the reflection at the mirror. 


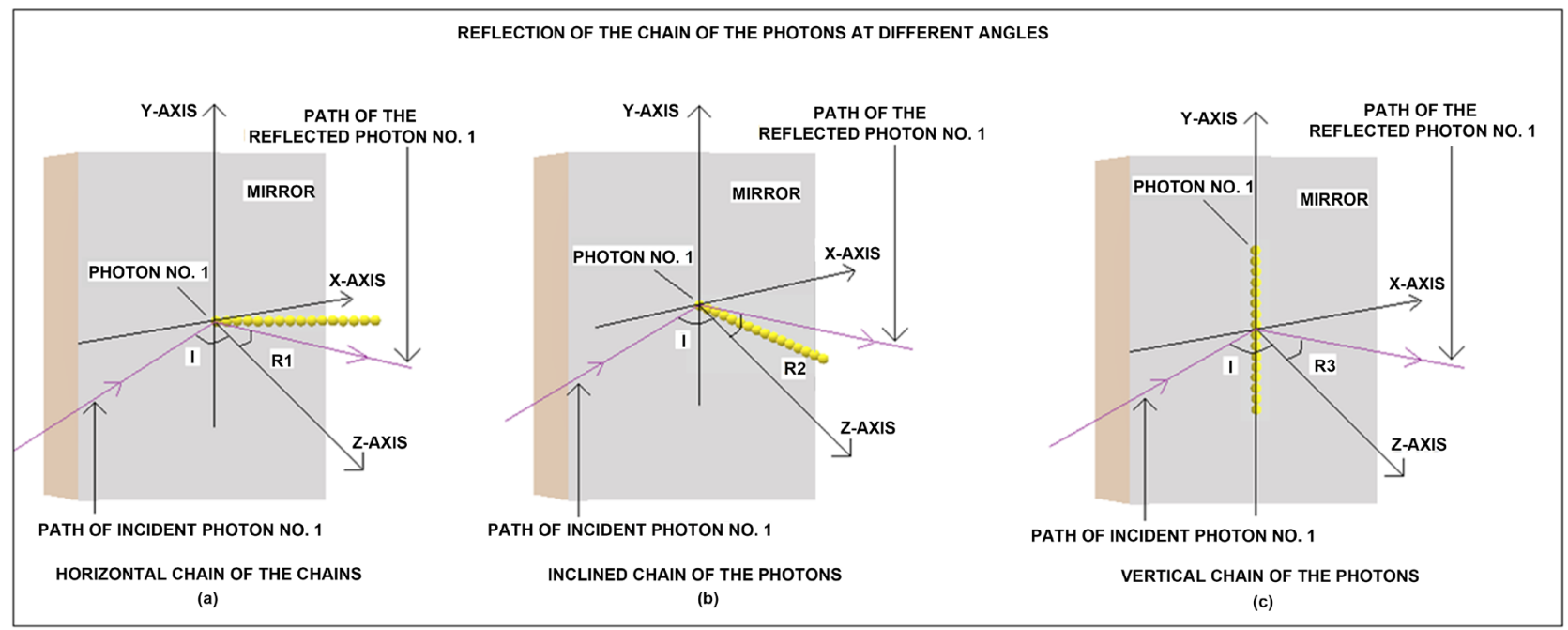

Figure 8. The reflection of the long chain of the photons on a vertical mirror. Figure 8(a) shows a horizontal chain of the photons reflecting on a mirror. Figure $8(\mathrm{~b})$ shows an inclined chain of the photons reflecting on a mirror. Figure 8 (c) shows a vertical chain of the photons reflecting on a mirror. All the figures are not to scale.

The most critical feature of any reflection experiment is the increase in the angle of deviation with the increase in the frequency of the photons under the same conditions. If the photons have zero mass, no inertial torque develops, and on reflection there is no deviation of the photons.

The deviation of the photons on reflection and the increase in the angle of deviation with the higher frequency photons prove the presence of a mass located off-center in the photons.

The following experiments prove and determine the mass, diameter \& charge of the photons:

\subsection{Multiple Slits in Series Experiment}

- A set of a single and a double slit creates constructive interference of the two photons and join them side by side. A typical frame mounts several sets of such single \& double slits in the series in perfect alignment with the facility to rotate all the slits together in $360^{\circ}$ angles.

- Every set of the slits doubles the number of the photons joined together side by side from 2 to 4,4 to 8,8 to 16,16 to 32 and so on by the constructive interference.

- When the same frequency photons join side by side, the strong force due to the mass in the photons is applied, and the photons join to produce a long chain of the photons spinning together.

- All the slits in the same vertical plane produce a horizontal chain of the spinning photons. The chain of the spinning photons forms in a line perpendicular to the plane of all the slits.

- As all the slits rotate, the angle of the chain of the spinning photons changes. With all the slits in the horizontal plane, the chain of photon forms in a vertical line. 
- A vertical plain surface coated mirror, in an inclined position, reflects the long chain of the spinning photons. A detector, installed at a distance, detects and records the position of the reflected chain of the photons and the changes in the angles of deviation in different directions.

- The above set up determines the mass, the charge and the diameter of the photons of different frequencies.

\subsection{To Determine the Mass of the Photons}

- An inertial torque develops on reflection of a chain of the spinning photons by the rotating mass in each photon of the chain. Figure 8(a) and Figure 8(b) show the chain of the spinning photons and the mirror. Only the first photon of the chain contacts the mirror and reflects along with the whole chain of the photons attached to the first photon.

- All the photons transfer the torques generated to the first photon. The first photon at the contact point of the mirror turns, due to all the inertial torques from all the photons, to deviate on reflection in the different directions.

- After the reflection, the angle of the whole chain of the spinning photons also changes in the different directions.

- The angles of the deviation depend on the angle of the chain of the spinning photons at the contact point of the mirror.

The following equations can calculate the mass of the photon:

- The angle of deviation in X-direction:

$$
\begin{aligned}
\Phi_{X}: & k m f\left[(r \sin \theta)^{2}+\{(D+r) \sin \theta\}^{2}+\{(2 D+r) \sin \theta\}^{2}\right. \\
& \left.+\cdots+\{(n D-D+r) \sin \theta\}^{2}\right]
\end{aligned}
$$

- The angle of deviation in Y-direction:

$$
\begin{aligned}
& \Phi_{Y}: k m f\left[(r \cos \theta)^{2}+\{(D+r) \cos \theta\}^{2}+\{(2 D+r) \cos \theta\}^{2}\right. \\
& \left.+\cdots+\{(n D-D+r) \cos \theta\}^{2}\right]
\end{aligned}
$$

where:

- $k$. a constant

- $\theta$. Polarisation angle of the photons

- $D$ : Diameter of the photon

- $n$ : Number of photons in the chain

A set of data generates by varying the angle of the chain of the spinning photons and the angle of incidence on the mirror to calculate the mass of the photon.

\subsection{To Determine the Charge of the Photons}

- The multiple slits produce a long chain of the photons joined together side by side in any required number by the constructive interference.

- All the photons are in the same phase in the chain of the spinning photons. 
As all the photons are close enough, the magnetic fields of all the photons combine to form a single peak of the strong magnetic field.

- A magnetic field detector detects and records the combined magnetic field.

- The division of the combined magnetic field by the number of the photons calculates the strength of the magnetic field of a single photon which in turn calculates the charge of the photon using already known equations.

\subsection{To Determine the Diameter of a Photon}

- All the multiple slits in the horizontal plane form a long vertical chain of the spinning photons. Figure 7 (c) shows a vertical chain of the photons with the mirror.

- A detector can detect both the ends of the chain of the photon after reflection or directly without reflection. The distance between the first and the last photon divided by the number of the photons calculates the diameter of the photon.

Note: The instruments with the required high precision are not available in the private laboratory of the author.

\section{Conclusions}

The essence of a photon/quantum particle is the presence of a nucleus of the mass and the charge, and the most critical feature is the off-center location of the nucleus in the particle. The intermediate interference decisively discovers and proves the presence of only one nucleus of the mass located off-center in the photons.

The nucleus of the mass and charge rotates around the center of the photon with the spin of the photon and continuously accelerates/decelerates in the different directions, and these accelerations of the mass in the nucleus generate the forces of varying magnitudes in the different directions. The continuously varying forces in the different directions move a photon as a wave and explain the Wave-Particle Duality pending since the year 1801. Similarly, the regular accelerations/decelerations of the charge in the nucleus explain the formation of an electromagnetic wave by a photon/ quantum particle.

During reflection of a photon, the rotating mass in the photon creates inertial torque at the contact point of the mirror in different directions. These inertial forces deviate the photon a little in different directions after the reflection. The increase in the frequency of the photons increases the angles of deviation in the different directions. Higher the frequency, the higher is the inertial torque developed by the mass of the photons. The generation of the inertial torque, at the contact point of the mirror, proves the presence of a mass located off-center in the photons.

All the quantum phenomena prove the presence of a nucleus of the mass and the charge located off-center in the photons/quantum particles as stated in the New Quantum Theory. The experiments using high precision instruments can 
determine the mass, the charge and the diameter of the photons.

\section{Conflicts of Interest}

The authors declare no conflicts of interest regarding the publication of this paper.

\section{References}

[1] Agarwal, N.S. (2012) Indian Journal of Science and Technology, 5, 3612-3617.

[2] Agarwal, N.S. (2016) Journal of Modern Physics, 7, 2135-2154.

https://doi.org/10.4236/jmp.2016.715186 